\title{
PROPRIEDADES FÍSICAS E QUÍMICAS DE UM ARGISSOLO SOB CULTIVO DE Eucalyptus dunnii Maiden NO PAMPA GAÚCHO ${ }^{1}$
}

\author{
PHYSICAL AND CHEMICAL PROPERTIES OF AN ULTISOL UNDER CULTIVATION OF \\ Eucalyptus dunnii Maiden OF PAMPA GAÚCHO
}

\author{
Suzana Ferreira da Rosa ${ }^{2}$ Dalvan José Reinert ${ }^{3}$ José Miguel Reichert ${ }^{3}$ Frederico Dimas Fleig ${ }^{4}$ \\ Miriam Fernanda Rodrigues ${ }^{4}$ Neiva Somavilla Gelain ${ }^{5}$
}

\begin{abstract}
RESUMO
A conversão de uma área de vegetação natural para uma área de cultivo associada às mudanças na cobertura vegetal podem acarretar modificações em algumas propriedades do solo, interferindo em sua qualidade. Dessa forma, o trabalho teve o objetivo de avaliar as alterações ocorridas nas propriedades físicas e químicas de um solo arenoso sob vegetação de campo nativo após a implantação de povoamentos de Eucalyptus dunnii Maiden, em diferentes densidades populacionais. $\mathrm{O}$ trabalho foi realizado na região da Campanha Gaúcha, município de São Francisco de Assis-RS. Foi instalado um experimento implantado com Eucalyptus dunnii Maiden sobre campo nativo, nos espaçamentos 3,50 m x 3,50 m; 3,50 m x 1,75 m; $1,75 \mathrm{~m}$ x 1,75 m e 1,75 m x 0,87 m, em um Argissolo Vermelho Distrófico. O delineamento experimental utilizado foi o de blocos ao acaso, com quatro repetições. Foram coletadas, em cada unidade experimental, amostras de solo nas profundidades de 0,00-0,10 m; 0,10-0,20 m e 0,20-0,40 m antes e após 12 meses da implantação. As propriedades físicas do solo avaliadas foram densidade, porosidade total, macroporosidade, microporosidade do solo e distribuição do tamanho dos agregados estáveis em água. As propriedades químicas determinadas foram: $\mathrm{pH} \mathrm{H}_{2} \mathrm{O}$, alumínio $(\mathrm{Al})$, cálcio $(\mathrm{Ca})$, magnésio $(\mathrm{Mg})$, e potássio $(\mathrm{K})$ trocáveis, fósforo disponível $(\mathrm{P})$, nitrogênio total $(\mathrm{N})$ e carbono orgânico total $(\mathrm{C})$. Ocorreram alterações significativas na macro e microporosidade, na porcentagem dos agregados pertencentes à classe de tamanho de 8,00-4,75 $\mathrm{mm}$ e no DMG devido às práticas de preparo do solo e do manejo do povoamento no período inicial. Para as propriedades químicas do solo, houve aumento no $\mathrm{pH}$ e nos níveis de Ca no solo após 12 meses devido ao efeito da adubação aplicada.
\end{abstract}

Palavras-chave: povoamentos florestais; qualidade do solo; espaçamento de plantio.

\begin{abstract}
The conversion of natural vegetation to cultivated areas as well as changes in vegetation cover can lead to changes in some soil properties, interfering on their quality. Such changes occur in the soil structure and in the chemical properties. Therefore, the study has the objective to evaluate the changes in the physical and chemical properties of an Ultisol after afforestation of Eucalyptus dunnii Maiden planted in different

1 Trabalho desenvolvido como parte da dissertação de mestrado apresentada ao PPG em Engenharia Florestal (UFSM) da primeira autora, com apoio do CNPq e do grupo Stora Enso.

2 Engenheira Florestal, Dr ${ }^{\mathrm{a}}$., Professora do Instituto Federal de Educação Ciência e Tecnologia do Rio Grande do Sul (IFRS), Rua Nelsi de Ribas Fritsch, 11111, Bairro Esperança, CEP 98200-000, Ibirubá (RS), Brasil. suzana. rosa@ibiruba.ifrs.edu.br

3 Engenheiro Agrônomo, Dr., Professor do Departamento de Solos, Centro de Ciências Rurais, Universidade Federal de Santa Maria, Av. Roraima, s/n, CEP 97105-900, Santa Maria (RS), Brasil. dalvan@ufsm.br / reichert. jm@googlemail.com

4 Engenheiro Florestal, Dr., Professor do Departamento de Ciências Florestais, Centro de Ciências Rurais, Universidade Federal de Santa Maria, Av. Roraima, s/n, CEP 97105-900, Santa Maria (RS), Brasil. dimasfleig@ uol.com.br / miriamf_rodrigues@yahoo.com.br

5 Engenheira Florestal, MSc., Programa de Pós-graduação em Engenharia Florestal, Centro de Ciências Rurais, Universidade Federal de Santa Maria, Av. Roraima, s/n, CEP 97105-900, Santa Maria (RS), Brasil. neivaengenheira@gmail.com
\end{abstract}

Recebido para publicação em 29/09/2011 e aceito em 16/03/2017

Ci. Fl., v. 28, n. 2, abr. - jun., 2018 
spacing resulting in several plant populations. The study was accomplished in 'Campanha Gaúcha' region, in São Francisco de Assis city, Rio Grande do Sul state (RS). It was carried out an experiment planted with Eucalyptus dunnii Maiden planted on Ultisol with native grass. The plant spacings used were: 3,50 m x $3,50 \mathrm{~m} ; 3,50 \mathrm{~m} \times 1,75 \mathrm{~m} ; 1,75 \mathrm{~m} 1,75 \mathrm{~m}$ and $1,75 \mathrm{~m} \times 0,87 \mathrm{~m}$. The experimental design was a complete randomized block with four replicates. It was collected soil samples were taken before the Eucalyptus planting and after twelve months, and layers of $0,00-0,10 \mathrm{~m} ; 0,10-0,20 \mathrm{~m}$ and $0,20-0,40 \mathrm{~m}$. The soil physical properties measured were: bulk density, total, macro and microporosity and wet aggregate size distribution. The soil chemical measured were: water $\mathrm{pH}, \mathrm{Al}^{+++}, \mathrm{Ca}^{++}, \mathrm{Mg}^{++}, \mathrm{K}^{+}$, available $\mathrm{P}, \mathrm{N}$ and $\mathrm{C}$. There were change in the macro and microporosity, in the percentage of stable aggregates of size class $8,00-4,75 \mathrm{~mm}$ and DMG because to soil tillage and forest management. For the chemical properties there were increase in the $\mathrm{pH}$ and Ca content in the soil after twelve months by effect to fertilization applied.

Keywords: forest stands; soil quality; plant spacing.

\section{INTRODUÇÃO}

O cultivo florestal com espécies de rápido crescimento tem se intensificado nos últimos anos no Rio Grande do Sul, tendo como a maior área de expansão situada no Bioma Pampa, onde ocorre a conversão de áreas usadas com pecuária extensiva, formada por vegetação de campo nativo, em povoamentos florestais homogêneos, em especial o eucalipto. No entanto, apesar da expansão de florestas homogêneas de eucalipto ocorrer em larga escala no Brasil e ocuparem extensas áreas, há poucos estudos a respeito dos impactos desses povoamentos no ambiente natural, sobretudo no solo (MARTINS et al., 2002), que apresenta restrições ao uso e manejo devido à fragilidade estrutural. Além disso, de acordo com estes autores, diferentes coberturas vegetais proporcionam distintos comportamentos em relação aos atributos do solo, sendo tais modificações em diferentes graus.

Desde as primeiras plantações no Brasil, o gênero Eucalyptus tem sido alvo de questionamentos e estudos a respeito do seu efeito nas modificações nos atributos do solo. De acordo com Lima (1996), as principais preocupações nesses estudos referem-se às possíveis alterações proporcionadas ao solo pela decomposição da serapilheira e pelo consumo de água pelos povoamentos florestais homogêneos formados com este gênero.

Alterações das propriedades físicas do solo podem manifestar-se de várias maneiras, como na alteração da estabilidade dos agregados, na densidade e na porosidade, redução na capacidade de armazenamento de água, na resistência à erosão hidráulica e eólica, com reflexos ambientais e influenciando o desenvolvimento das plantas (ARATANI et al., 2009). Na maioria dos solos florestais brasileiros, a adição de material orgânico tem se mostrado como uma técnica capaz de aumentar a agregação, a capacidade de armazenamento de água, a condutividade hidráulica, a densidade, a resistência à compactação e à erosão hidráulica e eólica (LEROY et al., 2008).

Quanto às propriedades químicas, as modificações de uso do solo alteram a dinâmica de nutrientes, através da demanda pelas plantas e a deposição e decomposição da matéria orgânica, a qual é um dos principais indicadores químicos do solo para avaliação das modificações promovidas pelo uso e ou manejo. Nos povoamentos florestais, o movimento dos nutrientes ocorre através da absorção pelas plantas de camadas mais profundas do solo, quando comparado a outros sistemas de cultivos, e o seu retorno pela liberação dos nutrientes do material vegetal depositado sobre o solo, por meio da decomposição microbiana (VEZZANI; TEDESCO; BARROS, 2001).

Diversos estudos já foram realizados para comparar os efeitos das práticas de manejo do solo e cultivos sobre a produção e rendimento das culturas (GAVANDE, 1976). No entanto, o impacto dos plantios florestais sobre o solo depende do local em que este está inserido, devendo ser realizados estudos detalhados e localizados em áreas cultivadas com o eucalipto para se ter um real diagnóstico do efeito da espécie na qualidade do solo.

Além disso, há estudos que relacionam diferentes usos do solo de acordo com sua qualidade, a exemplo disso, Alvarenga (1996), estudando a alteração de atributos de solo sob diferentes condições de uso, observou, por meio de análise de componentes principais, que os atributos do solo mais correlacionados com as alterações nos ecossistemas foram os teores de $\mathrm{K}, \mathrm{Mg}, \mathrm{Ca}$ e $\mathrm{S}$, a densidade do solo, a porosidade 
total, a macroporosidade e a percentagem de agregados superiores a $2 \mathrm{~mm}$. Melloni et al. (2008), em estudo avaliando a qualidade do solo sob diferentes coberturas florestais e pastagem, verificaram que as propriedades químicas do solo mais sensíveis ao uso foram os teores de $\mathrm{C}, \mathrm{Ca}, \mathrm{K}, \mathrm{Mn}, \mathrm{B}$, saturação por bases, Al trocável e saturação por Al. Evidenciando o efeito do uso do solo em algumas propriedades químicas.

Além do uso do solo, as práticas de manejo adotadas também interferem na sua qualidade. Como exemplo disso, na área florestal, o espaçamento de plantio muito provavelmente vai determinar a demanda nutricional do povoamento, podendo promover um empobrecimento químico do solo em espaçamentos muito adensados. Por outro lado, os espaçamentos mais densos podem melhorar a agregação do solo e iniciar o armazenamento de carbono mais cedo quando comparado com espaçamentos amplos devido ao fechamento precoce das copas promovendo uma maior deposição de serapilheira e uma maior densidade de raízes.

O espaçamento entre plantas em um sítio florestal deve ser determinado em função do grau de limitação da área, como disponibilidade de água e de nutrientes no solo. Além disso, a escolha do espaçamento também deve considerar as espécies, as quais apresentam diferentes comportamentos dependendo do local e do objetivo de produção, ou seja, o uso futuro da madeira a ser produzida (TONINI, 2003). As diferentes densidades populacionais dos povoamentos florestais podem levar a diferentes quantidades de nutrientes removidos do solo, pois, quanto menor a densidade, mais intensa será a atividade do sistema radicular em maiores profundidades, aumentando a quantidade de nutrientes disponíveis por planta (LEITE et al., 1998). Desta forma, as menores densidades populacionais podem levar a uma redução de crescimento já em povoamentos jovens, pois ocorre uma maior exaustão de nutrientes do solo e um menor acúmulo de nutrientes por planta.

Os questionamentos a respeito dos efeitos dos povoamentos florestais homogêneos de eucaliptos nas propriedades do solo e do grau de limitação oferecido pelos solos arenosos na região sudoeste do Rio Grande do Sul foram os fatores que nortearam a realização do presente estudo. Dessa forma, o objetivo do estudo foi verificar as alterações físicas e químicas ocorridas em um Argissolo Vermelho Distrófico com o plantio de Eucalyptus dunnii em diferentes densidades populacionais.

\section{MATERIAIS E MÉTODOS}

O trabalho foi realizado em uma área experimental, no município de São Francisco de Assis. A região de estudo faz parte da campanha sudoeste do Rio Grande do Sul, situada no bioma Pampa, onde ocorre a expansão do cultivo de espécies florestais de rápido crescimento, especialmente de eucalipto, para a produção de madeira para celulose. A área de instalação do experimento localiza-se na Fazenda Cerro

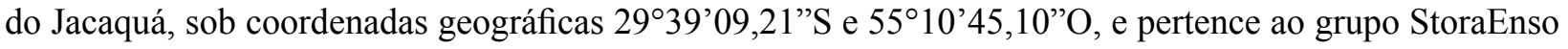
divisão Rio Grande do Sul.

O clima da região, segundo a classificação de Köppen (MORENO, 1961), é do tipo Cfa, com temperatura média anual de $18^{\circ} \mathrm{C}$. O solo da área em estudo é classificado como Argissolo Vermelho Distrófico (STRECK et al., 2008).

O experimento foi instalado em uma área de campo nativo, com vegetação natural formada por herbáceas, anteriormente usada para o pastejo bovino.

Os tratamentos foram compostos por diferentes espaçamentos de plantio de Eucalyptus dunnii Maiden: 3,50 m x3,50 m; 3,50 m x 1,75 m; $1,75 \mathrm{~m} \mathrm{1,75} \mathrm{m} \mathrm{e} \mathrm{1,75} \mathrm{m} \mathrm{x} \mathrm{0,87} \mathrm{m,} \mathrm{sendo} \mathrm{o} \mathrm{número} \mathrm{de} \mathrm{plantas}$ por hectare de cada espaçamento: 816, 1632, 3265 e 6568 árvores/ha, respectivamente. O delineamento experimental utilizado foi o de blocos ao acaso com quatro repetições.

O preparo do solo foi do tipo reduzido, sendo realizado por meio de escarificação mecânica na linha de plantio, a profundidade do sulco foi de $0,30 \mathrm{~m}$ e foi aplicado $200 \mathrm{~kg} /$ ha de hiperfosfato natural reativo no sulco, na formulação de $29 \%$ de $\mathrm{P}_{2} \mathrm{O}_{5}, 10 \% \mathrm{P}_{2} \mathrm{O}_{5}$ em ácido cítrico e $36 \%$ de cálcio. A vegetação de campo nativo foi suprimida com a aplicação do herbicida Glifosato na dose de $1,2 \mathrm{~kg} / \mathrm{ha}$. O plantio das mudas foi realizado manualmente e ocorreu em outubro de 2008, logo após as operações de preparo do solo. Na condução do experimento foi realizada uma adubação de cobertura após um mês do plantio, na qual foi aplicado $100 \mathrm{~g}$ de NPK (6:30:6) por planta, em covas laterais, distanciadas $15 \mathrm{~cm}$ das mudas. 
A coleta de solo foi realizada em um ponto dentro de cada unidade experimental, anteriormente e após 12 meses do plantio das mudas. Sendo constituída, para cada parcela uma amostra simples. As amostras de solo foram coletadas em trincheiras, nas camadas 0-0,10 m; 0,10-0,20 $\mathrm{m}$ e 0,20-0,40 m.

A granulometria e a densidade das partículas do solo foram determinadas nas amostras coletadas antes das operações do plantio. Para a determinação granulométrica foi utilizado o método da pipeta (EMBRAPA, 1997), sendo utilizado como dispersante o NaOH. Na Tabela 1 observa-se a distribuição granulométrica das partículas do solo nas camadas estudadas e classificação da textura do solo nas camadas, segundo o triangulo para a classificação das classes texturais adotado pela Sociedade Brasileira de Ciência do Solo.

TABELA 1: Caracterização granulométrica das camadas do Argissolo Vermelho Distrófico na região de São Francisco de Assis-RS, Brasil.

TABLE 1: $\quad$ Size grain of Ultisol in the study area in São Francisco de Assis, RS state, Brazil.

\begin{tabular}{cccccc}
\hline Camada & Areia Grossa & Areia Fina & Silte & Argila & \\
\cline { 2 - 5 }$(\mathrm{m})$ & & $\left(\mathrm{g} \mathrm{kg}^{-1}\right)$ & & & Classe Textural \\
\hline $0,00-0,10$ & 246 & 600 & 91 & 62 & Areia \\
$0,10-0,20$ & 226 & 571 & 100 & 102 & Areia Franca \\
$0,20-0,40$ & 217 & 553 & 110 & 119 & Areia Franca \\
\hline
\end{tabular}

As propriedades físicas do solo analisadas foram: densidade do solo, porosidade total, macroporosidade, microporosidade e o diâmetro médio geométrico (DMG) dos agregados estáveis em água. A densidade foi determinada pelo método do anel volumétrico (EMBRAPA, 1997). A porosidade total foi determinada pela relação entre a densidade do solo e a densidade das partículas (EMBRAPA, 1997), enquanto a microporosidade foi determinada em amostras com estrutura preservada, nas quais os cilindros metálicos, contendo as amostras, foram submetidos à saturação por 24 horas e colocados em mesa de tensão com $60 \mathrm{~cm}$ de altura de coluna de água $(6 \mathrm{kPa})$. A macroporosidade foi calculada pela diferença da porosidade total e microporosidade. A densidade das partículas foi determinada pelo método do balão volumétrico modificado (GUBIANI et al., 2006). A distribuição do tamanho dos agregados estáveis em água foi determinada segundo a metodologia de Kemper e Chepil (1965), sendo utilizadas as peneiras de tamiz 4,75, 2,00, 1,00 e $0,21 \mathrm{~mm}$.

Nas determinações das propriedades químicas do solo, foram realizadas análise de $\mathrm{pH}$ em água; teores de fósforo disponível $(\mathrm{P})$ e de potássio $(\mathrm{K})$, cálcio $(\mathrm{Ca})$, magnésio $(\mathrm{Mg})$ e alumínio $(\mathrm{Al})$ trocáveis, carbono orgânico total $(\mathrm{C})$ e nitrogênio total $(\mathrm{N})$. Essas determinações químicas seguiram Tedesco et al. (1995), sendo utilizado o extrator Mehlich 1 para o $\mathrm{P}$ e o $\mathrm{K}$, o extrator $\mathrm{KCl}$ para o $\mathrm{Ca}, \mathrm{Mg}$ e $\mathrm{Al}$, enquanto o $\mathrm{C}$ e o N foram determinados em Analisador Elementar modelo FlashEA 1112.

Os resultados foram submetidos à comparação de médias dos dados obtidos antes e após os 12 meses da implantação do eucalipto pelo teste " $t$ " de Student para dados independentes, comparando os dados dentro de cada tratamento.

\section{RESULTADOS E DISCUSSÃO}

Não houve efeito dos espaçamentos de plantio nas propriedades físicas do solo (Tabela 2). O plantio de eucalipto não promoveu alterações significativas na densidade do solo até o primeiro ano de cultivo (Tabela 2). Os valores de densidade, nas distintas camadas, variaram de $1,44 \mathrm{Mg} \mathrm{m}^{-3}$ a $1,66 \mathrm{Mg} \mathrm{m}^{-3}$, e estão situados entre os limites de densidades de solos de textura areia e franco-arenosa, que podem ter uma variação entre 1,20 até $1,80 \mathrm{Mg} \mathrm{m}^{-3}$ (BRADY, 1989). Dessa forma, através da análise dos valores da densidade do solo, pode-se inferir que as operações de preparo do solo e de manutenção do povoamento efetuadas no primeiro ano não alteraram a densidade do solo em níveis limitantes ao desenvolvimento normal das plantas (REICHERT et al., 2009), nem mesmo para os espaçamentos mais densos, nos quais ocorreu maior intensidade de tráfego. Os valores de densidade mostraram-se inferiores aos níveis críticos ou restritivos, de acordo com as estimativas propostas por Reichert, Suzuki e Reinert (2007), que relacionam a densidade e o teor de argila do solo. Os limites críticos restritivos propostos pelos autores são de 1,91; 1,93 e $1,94 \mathrm{Mg} \mathrm{m}^{-3}$, para as camadas $0,0-0,10 \mathrm{~m} ; 0,10-0,20 \mathrm{~m}$ e $0,20-0,40 \mathrm{~m}$, respectivamente. 
Não se observou diferença significativa para a porosidade total antes e após 12 meses de implantação do eucalipto nos distintos espaçamentos de plantio. Para a microporosidade houve aumento significativo para o espaçamento $3,50 \times 1,75 \mathrm{~m}$, nas camadas $0,00-0,10 \mathrm{~m}$. Na camada $0,20-0,40 \mathrm{~m}$ ocorreu redução da macroporosidade e aumento da microposidade 12 meses após a implantação do eucalipto nos espaçamentos $3,50 \times 3,50 \mathrm{~m}, 3,50 \times 1,75 \mathrm{~m}$ e $1,75 \times 0,87 \mathrm{~m}$.

A redução da macroporosidade e aumento da microporosidade na camada $0,20-0,40 \mathrm{~m}$ pode ser reflexo da compactação do solo, em função do tráfego durante as operações de preparo do solo e condução do povoamento. A compactação não se manifestou nas camadas superficiais, possivelmente, devido a uma maior compactação preexistente das partículas do solo, em função do pastejo exercido anteriormente na área ou devido à maior presença de material orgânico e à atividade biológica e radicular sobre a camada superficial (PREVEDELLO, 2008; REICHERT et al., 2009). O efeito da atividade biológica é menos pronunciado em profundidade, proporcionando densidades do solo mais elevadas.

Os resultados do aumento da microporosidade corroboram os estudos de Reichert, Suzuki e Reinert (2007), ao afirmarem que o tráfego de máquinas pode proporcionar a redução na proporção dos poros maiores, responsáveis pela aeração do solo, que são substituídos por poros menores, principalmente poros que retêm água. Dessa forma, a redução da macroporosidade com aumento da microporosidade do solo em estudo, ocorrida na camada $0,20-0,40 \mathrm{~m}$, pode ser considerada, até certos limites, benéfica, pois proporciona maior capacidade de retenção e disponibilidade da água neste tipo de solo que apresenta uma elevada porosidade com rápida infiltração da água.

Avaliando coberturas florestais e de pastagem na qualidade dos solos no sul de Minas Gerais, Melloni et al. (2008), observaram que a microporosidade não diferiu entre os sistemas estudados, enquanto que a macroporosidade foi maior na mata $(34,31 \%$ ), diferindo dos demais, cujos valores foram de $20,81 \%$, $16,52 \%$ e 13,53 \% para eucalipto, araucária e pastagem, respectivamente. Nesse caso, a macroporosidade foi mais sensível ao uso do solo, enquanto no presente estudo, a microporosidade demonstrou maior sensibilidade à mudança de uso do solo.

TABELA 2: Densidade, Porosidade total, microporosidade e macroporosidade do Argissolo Vermelho Distrófico nos espaçamentos 3,50 x 3,50 m (E1), 3,50 x 1,75 m (E2), 1,75 x 1,75 m (E3) e 1,75 x 0,87 m (E4) de Eucalyptus dunnii Maiden antes (AP) e após um ano de implantação (DP), São Francisco de Assis-RS, Brasil.

TABLE 2: Bulk density, total, micro and macroporosity of Ultisol planted by Eucalyptus dunnii Maiden on 3,50 x 3,50 m (E1), 3,50 x 1,75 m (E2), 1,75 x 1,75 m (E3) e 1,75 x 0,87 m (E4) spacing before and after 12 months planted, São Francisco de Assis, RS state, Brazil.

\begin{tabular}{|c|c|c|c|c|c|c|c|c|}
\hline Camada (m) & E1 AP & E1 DP & E2 AP & E2 DP & E3 AP & E3 DP & E4 AP & E4 DP \\
\hline \multicolumn{9}{|c|}{ Densidade do solo $\left(\mathrm{Mg} \mathrm{m}^{-3}\right)$} \\
\hline $0,00-0,10$ & 1,58 & 1,58 & 1,56 & 1,59 & 1,60 & 1,59 & 1,57 & 1,58 \\
\hline $0,10-0,20$ & 1,66 & 1,63 & 1,61 & 1,58 & 1,64 & 1,60 & 1,64 & 1,65 \\
\hline $0,20-0,40$ & 1,56 & 1,57 & 1,59 & 1,58 & 1,55 & 1,57 & 1,58 & 1,57 \\
\hline \multicolumn{9}{|c|}{ Porosidade Total $\left(\mathrm{m}^{3} \mathrm{~m}^{-3}\right)$} \\
\hline $0,00-0,10$ & 0,397 & 0,399 & 0,400 & 0,389 & 0,379 & 0,382 & 0,389 & 0,388 \\
\hline $0,10-0,20$ & 0,364 & 0,375 & 0,380 & 0,390 & 0,370 & 0,383 & 0,369 & 0,376 \\
\hline $0,20-0,40$ & 0,401 & 0,400 & 0,388 & 0,390 & 0,408 & 0,405 & 0,397 & 0,403 \\
\hline \multicolumn{9}{|c|}{ Macroporosidade $\left(\mathrm{m}^{3} \mathrm{~m}^{-3}\right)$} \\
\hline $0,00-0,10$ & 0,218 & 0,190 & 0,226 & 0,177 & 0,204 & 0,185 & 0,215 & 0,196 \\
\hline $0,10-0,20$ & 0,187 & 0,174 & 0,199 & 0,191 & 0,200 & 0,194 & 0,194 & 0,179 \\
\hline $0,20-0,40$ & 0,216 & 0,179 & $0,206^{*}$ & $0,165^{*}$ & 0,232 & 0,209 & $0,218^{*}$ & $0,180^{*}$ \\
\hline \multicolumn{9}{|c|}{ Microporosidade $\left(\mathrm{m}^{3} \mathrm{~m}^{-3}\right)$} \\
\hline $0,00-0,10$ & 0,179 & 0,209 & $0,174 *$ & $0,212 *$ & 0,174 & 0,197 & 0,174 & 0,192 \\
\hline $0,10-0,20$ & 0,177 & 0,201 & 0,181 & 0,199 & 0,171 & 0,189 & 0,175 & 0,197 \\
\hline $0,20-0,40$ & $0,184 *$ & $0,221 *$ & $0,182 *$ & $0,225 *$ & 0,175 & 0,196 & $0,1783 *$ & $0,2224 *$ \\
\hline
\end{tabular}

Em que: *Diferença significativa antes e após um ano de implantação do Eucalyptus dunnii Maiden dentro de cada tratamento pelo teste t a $5 \%$ de probabilidade de erro.

A distribuição do tamanho dos agregados estáveis em água, na primeira camada, mostrou maior proporção de agregados pertencentes à classe de diâmetro de 8,00-4,75 mm (Tabela 3). Essa diferença ocorrida entre as duas camadas de solo pode estar relacionada ao efeito proporcionado pelo sistema radicular das gramíneas que faziam parte da vegetação de campo existente antes da implantação do eucalipto, o que 
contribuiu para a formação de agregados mais estáveis na camada superficial do solo. Resultado semelhante foi encontrado por Lima et al. (2008), estudando os atributos físicos de um Planossolo Háplico sob diferentes sistemas de manejo e comparando-os com área de campo nativo. Nesse trabalho, os autores observaram uma maior agregação do solo para as camadas superficiais na área de campo nativo, o qual apresentou a maior porcentagem dos agregados estáveis em água nas classes de tamanho de 8,00-4,76 mm. Da mesma forma, Flores et al. (2008) observaram maior quantidade de agregados na classe de 8,00-4,76 mm no campo nativo pastejado, 0,10 a $0,15 \mathrm{~m}$ e 0,20 a $0,25 \mathrm{~m}$ quando comparado com os sistemas mata nativa e cultivos agrícolas em plantio direto e convencional.

Um dos fatores responsáveis pela ocorrência de maior porcentagem dos agregados na classe entre 8-4,75 mm, principalmente na primeira camada de solo, é a ação dos agentes cimentantes. Segundo Tisdall e Oades (1982), agregados maiores são formados pela ação da matéria orgânica e pelo efeito das raízes. Dessa forma, a estabilização dos agregados do solo pode ter como agente principal a matéria orgânica e as raízes da vegetação competidora e de campo, pois o solo é arenoso, e os principais agentes estabilizantes neste caso são os fatores bióticos, como a matéria orgânica, os micro-organismos do solo e o sistema radicular (BAVER; GARDNER; GARDNER, 1972; WOHLENBERG et al., 2004).

Na comparação da distribuição do tamanho dos agregados estáveis em água antes e após 12 meses de implantação do Eucalyptus dunnii M. na camada 0,00-0,10 m, houve diferença significativa para o espaçamento 3,50 x 3,50 m nas classes de tamanho de 2,00-1,00 mm, para o espaçamento $1,75 \times 1,75 \mathrm{~m}$ nas classes 4,75-2,00 mm e 2,00-1,00 mm, e no espaçamento 1,75 x 0,87 m nas classes $8,00-4,75 \mathrm{~mm}$; $2,00-1,00 \mathrm{~mm}$ e $<0,21 \mathrm{~mm}$. Somente na classe $8,00-4,75 \mathrm{~mm}$, a agregação foi superior antes do plantio, enquanto nas demais classes e espaçamentos a porcentagem dos agregados estáveis em água foi superior após o plantio.

Em estudo realizado com o objetivo de determinar a influência de sistemas de culturas, de cobertura do solo e do teor de matéria orgânica sobre a agregação de um Argissolo Vermelho-Amarelo Distrófico arênico, ao longo do tempo, Wohlenberg et al. (2004) observaram que, independentemente da época de amostragem, os maiores valores de DMG sempre ocorreram no campo natural, mesmo com níveis de fertilidade inferiores aos dos sistemas agrícolas com adição de fertilizantes químicos. Evidenciando o efeito benéfico do sistema radicular da vegetação de campo na formação dos agregados de maiores tamanhos e mais estáveis. Avaliando a recuperação da qualidade estrutural, pelo sistema plantio direto, de um Argissolo Vermelho, Flores et al. (2008) observaram que o cultivo do solo em relação aos sistemas campo e mata reduziu a quantidade de agregados maiores e aumentou a dos menores.

TABELA 3: Porcentagem dos agregados estáveis em água nas camadas 0,00-0,10 m e 0,10-0,20 m do Argissolo Vermelho Distrófico nos espaçamentos 3,50 x 3,50 m (E1), 3,50 x 1,75 m (E2), 1,75 x 1,75 m (E3) e 1,75 x 0,87 m (E4) de Eucalyptus dunnii Maiden antes (AP) e após (DP) um ano de implantação, São Francisco de Assis-RS, Brasil.

TABLE 3: Rate wet aggregate size distribution on the layers $0,00-0,10 \mathrm{~m} \mathrm{e} 0,10-0,20 \mathrm{~m}$ of Ultisol on the planting spacing 3,50 x 3,50 m (E1), 3,50 x 1,75 m (E2), 1,75 x 1,75 m (E3) e 1,75 x 0,87 m (E4) of Eucalyptus dunnii Maiden before (AP) and after (DP) 12 months planted, São Francisco de Assis, RS state, Brazil.

\begin{tabular}{|c|c|c|c|c|c|c|c|c|c|c|c|c|}
\hline \multicolumn{7}{|c|}{ Antes plantio } & \multicolumn{6}{|c|}{ Após o plantio } \\
\hline Trat. & $8-4,75$ & $4,75-2$ & $2-1$ & $1-0,21$ & $<0,21$ & $\begin{array}{l}\begin{array}{l}\text { DMG } \\
(\mathrm{mm})\end{array} \\
\end{array}$ & $8-4,75$ & $4,75-2$ & $2-1$ & $1-0,21$ & $<0,21$ & $\begin{array}{l}\text { DMG } \\
(\mathrm{mm})\end{array}$ \\
\hline \multicolumn{13}{|c|}{ Camada $0,00-0,10 \mathrm{~m}$} \\
\hline $3,50 \times 3,50$ & 90,68 & 4,95 & $0,31 *$ & $0,50 *$ & 3,55 & 5,26 & 82,29 & 9,5 & $0,69 *$ & $1,56^{*}$ & 5,95 & 4,57 \\
\hline $3,50 \times 1,75$ & 86,99 & 4,26 & 0,53 & 1,30 & 6,92 & 4,55 & 79,79 & 9,69 & 0,85 & 1,49 & 8,18 & 4,13 \\
\hline $1,75 \times 1,75$ & 88,84 & $4,47^{*}$ & $0,38^{*}$ & 1,24 & 5,06 & 4,90 & 79,43 & $11,18^{*}$ & $0,68^{*}$ & 1,97 & 6,74 & 4,31 \\
\hline $1,75 \times 0,87$ & $88,51^{*}$ & 5,43 & $0,42 *$ & 0,64 & $4,99 *$ & $4,91 *$ & 78,95 & $9,3^{*}$ & $1,05^{*}$ & 2,01 & $8,67 *$ & $3,97 *$ \\
\hline \multicolumn{13}{|c|}{ Camada $0,10-0,20 \mathrm{~m}$} \\
\hline $3,50 \times 3,50$ & 77,09 & 10,44 & 1,44 & 2,32 & 8,71 & $3,92 *$ & 55,21 & 13,34 & 3,48 & 12,15 & 15,8 & $2,37 *$ \\
\hline $3,50 \times 1,75$ & $88,61^{*}$ & $5,44^{*}$ & $0,65^{*}$ & 1,71 & 5,59 & 4,68 & $66,56^{*}$ & $12,75^{*}$ & $2,94 *$ & 7,05 & 10,69 & 3,26 \\
\hline $1,75 \times 1,75$ & 89,06 & $3,62 *$ & 0,32 & 0,87 & 6,12 & 4,76 & 66,05 & $13,52 *$ & 2,97 & 6,12 & 11,34 & 3,28 \\
\hline $1,75 \times 0,87$ & $87,8^{*}$ & $5,42 *$ & $0,53 *$ & 1,44 & $4,80 *$ & $4,86^{*}$ & $55,7 *$ & $14,21 *$ & $2,97 *$ & 9,62 & $17,49 *$ & $2,26^{*}$ \\
\hline
\end{tabular}

Em que: *Diferença significativa antes e após um ano de implantação do Eucalyptus dunnii Maiden dentro de cada tratamento pelo teste $\mathrm{t}$ a $5 \%$ de probabilidade de erro. 
$\mathrm{Na}$ distribuição dos agregados estáveis em água nas camadas de solo $0,10-0,20 \mathrm{~m}$, a porcentagem dos agregados estáveis em água foi significativamente menor nas classes 8,00-4,75 mm após 12 meses de implantação do eucalipto nos espaçamentos 3,50 x 1,75 m e 1,75 x 0,87 m. Suzuki (2008) observou aumento no DMG em área pastagem, cujo aumento foi atribuído à matéria orgânica e à compressão do solo pelo pisoteio animal.

As propriedades químicas do solo, antes da implantação de Eucalyptus dunnii M. e após 12 meses, estão apresentadas na Tabela 4. Diferenças significativas no $\mathrm{pH}$ e nos níveis de cálcio, magnésio e fósforo no solo foram observadas quando comparadas com a condição química antes e após 12 meses de implantação do Eucalyptus dunnii M. Observou-se aumento no pH do solo 12 meses após a implantação, cujas alterações foram significativas nas camadas $0,00-0,10 \mathrm{~m}$ para os espaçamentos $3,50 \times 1,75 \mathrm{~m}$ e 1,75 x 0,87 m, nas camadas $0,10-0,20 \mathrm{~m}$ nos espaçamentos $3,50 \times 3,50 \mathrm{~m} ; 3,50 \times 1,75 \mathrm{~m}$ e $1,75 \times 1,75 \mathrm{~m}$, e nas camadas $0,20-$ $0,40 \mathrm{~m}$ os espaçamentos $3,50 \times 3,50 \mathrm{~m} ; 3,50 \times 1,75 \mathrm{~m}$ e 1,75 x 0,87 m. Aumento no pH do solo em cultivos de eucalipto foram relatados Garay et al. (2004) quando comparados povoamentos Eucalyptus grandis com de Acacia mangium. Os autores justificam esse resultado pela capacidade de acidificação do solo que plantas fixadoras de $\mathrm{N}_{2}$ possuem. Considerando a grande presença de leguminosas em vegetação de campo nativo, pode-se mencionar o seu efeito nos baixos valores de $\mathrm{pH}$ do solo observados antes do plantio do eucalipto.

A liberação de exsudatos ácidos pelas raízes da vegetação de campo também pode ser um fator responsável pelos maiores valores de pH na condição anterior ao plantio (BARRETO et al., 2006).

O alumínio trocável do solo não foi alterado com a implantação do eucalipto nos espaçamentos de plantio utilizados, não sendo possível uma análise sobre o comportamento dessa variável no solo com a instalação de povoamentos de eucaliptos 12 meses após o plantio.

Com base nas variáveis que expressam a acidez, pode-se considerar o solo como muito ácido, cuja acidez, expressa pelo $\mathrm{pH}$, foi reduzida em 12 meses com a instalação do povoamento de Eucalyptus dunnii M. Também cabe ressaltar que não foi realizada calagem no solo, portanto, essa redução da acidez não apresenta aparente explicação para tal resultado.

Os valores de Ca sofreram acréscimos em todos os espaçamentos utilizados para todas as camadas de solo após 12 meses da implantação do eucalipto, tendo a adubação do solo, aumentado as concentrações do nutriente.

TABELA 4: Propriedades químicas do Argissolo Vermelho Distrófico nos espaçamentos 3,50 x 3,50 m (E1), 3,50 x 1,75 m (E2), 1,75 x 1,75 m (E3) e 1,75 x 0,87 m (E4) de Eucalyptus dunnii Maiden antes (AP) e após um ano de implantação (DP), São Francisco de Assis-RS, Brasil.

TABLE 4: Chemical properties of Ultisol planted with Eucalyptus dunnii Maiden in 3,50 x 3,50 m (E1), 3,50 x $1,75 \mathrm{~m}$ (E2), $1,75 \times 1,75 \mathrm{~m}$ (E3) e 1,75 x 0,87 m (E4) spacing before (AP) and after (DP) 12 months of planted, São Francisco de Assis, RS, Brazil.

\begin{tabular}{|c|c|c|c|c|c|c|c|c|}
\hline Camada (m) & E1 AP & E1 DP & E2 AP & E2 DP & E3 AP & E3 DP & E4 AP & E4DP \\
\hline \multicolumn{9}{|c|}{$\mathrm{pH}$} \\
\hline $0,00-0,10$ & 4,50 & 4,80 & $4,37^{*}$ & 4,82 & 4,67 & 4,90 & $4,49^{*}$ & 4,90 \\
\hline $0,10-0,20$ & $4,19^{*}$ & 4,70 & $4,12 *$ & 4,72 & $4,35^{*}$ & 4,72 & 4,30 & 4,70 \\
\hline $0,20-0,40$ & $3,99 *$ & 4,47 & $3,98^{*}$ & 4,47 & 4,27 & 4,57 & $3,94 *$ & 4,52 \\
\hline \multicolumn{9}{|c|}{$\mathrm{Al}\left(\mathrm{cmol}_{\mathrm{c}} \mathrm{dm}^{-3}\right)$} \\
\hline $0,00-0,10$ & 0,425 & 0,475 & 0,600 & 0,500 & 0,400 & 0,475 & 0,320 & 0,500 \\
\hline $0,10-0,20$ & 0,085 & 0,083 & 0,120 & 0,090 & 0,088 & 0,090 & 0,103 & 0,093 \\
\hline $0,20-0,40$ & 0,147 & 0,125 & 0,145 & 0,145 & 0,162 & 0,123 & $0,185^{*}$ & 0,128 \\
\hline \multicolumn{9}{|c|}{$\mathrm{Ca}\left(\mathrm{cmol}_{\mathrm{c}} \mathrm{dm}^{-3}\right)$} \\
\hline $0,00-0,10$ & $0,167^{*}$ & 0,800 & $0,197 *$ & 0,775 & $0,190 *$ & 0,625 & $0,070^{*}$ & 0,650 \\
\hline $0,10-0,20$ & $0,178^{*}$ & 0,725 & $0,155^{*}$ & 0,775 & $0,276^{*}$ & 0,750 & $0,098 *$ & 0,750 \\
\hline $0,20-0,40$ & $0,144 *$ & 0,650 & $0,148 *$ & 0,725 & $0,196^{*}$ & 0,750 & $0,070 *$ & 0,675 \\
\hline \multicolumn{9}{|c|}{$\mathrm{Mg}\left(\mathrm{cmol}_{\mathrm{c}} \mathrm{dm}^{-3}\right)$} \\
\hline $0,00-0,10$ & 0,230 & 0,275 & 0,260 & 0,300 & 0,251 & 0,250 & 0,199 & 0,250 \\
\hline $0,10-0,20$ & 0,266 & 0,300 & 0,190 & 0,300 & 0,190 & 0,275 & $0,184 *$ & 0,275 \\
\hline $0,20-0,40$ & 0,166 & 0,225 & $0,154 *$ & 0,250 & 0,158 & 0,300 & $0,136^{*}$ & 0,250 \\
\hline
\end{tabular}

Continuação... 
TABELA 4: Continuação...

TABLE 4: Continued..

\begin{tabular}{|c|c|c|c|c|c|c|c|c|}
\hline Camada (m) & E1 AP & E1 DP & E2 AP & E2 DP & E3 AP & E3 DP & E4 AP & E4DP \\
\hline \multicolumn{9}{|c|}{$\mathrm{P}\left(\mathrm{mg} \mathrm{dm}^{-3}\right)$} \\
\hline $0,00-0,10$ & 8,80 & 11,27 & 8,30 & 5,32 & 8,65 & 5,10 & $7,83^{*}$ & 4,50 \\
\hline $0,10-0,20$ & 4,35 & 3,55 & 4,04 & 3,35 & 4,54 & 3,00 & 5,19 & 2,22 \\
\hline $0,20-0,40$ & 3,07 & 2,40 & 3,00 & 2,05 & 2,68 & 2,05 & 2,93 & 1,67 \\
\hline \multicolumn{9}{|c|}{$\mathrm{K}\left(\mathrm{mg} \mathrm{dm}^{-3}\right)$} \\
\hline $0,00-0,10$ & 86,0 & 75,0 & 67,5 & 95,0 & 115,5 & 127,0 & 117,0 & 116,0 \\
\hline $0,10-0,20$ & 63,0 & 76,0 & 64,5 & 74,0 & 109,0 & 96,0 & 99,5 & 93,0 \\
\hline $0,20-0,40$ & 50,5 & 58,0 & 43,5 & 64,0 & 60,5 & 66,0 & 54,5 & 82,0 \\
\hline \multicolumn{9}{|c|}{$\mathrm{N}\left(\mathrm{g} \mathrm{kg}^{-1}\right)$} \\
\hline $0,00-0,10$ & 0,60 & 0,55 & 0,64 & 0,54 & 0,62 & 0,55 & 0,61 & 0,52 \\
\hline $0,10-0,20$ & 0,49 & 0,46 & 0,52 & 0,48 & 0,48 & 0,51 & 0,47 & 0,46 \\
\hline $0,20-0,40$ & 0,51 & 0,45 & 0,49 & 0,49 & 0,47 & 0,47 & 0,45 & 0,46 \\
\hline \multicolumn{9}{|c|}{$\mathrm{C}\left(\mathrm{g} \mathrm{kg}^{-1}\right)$} \\
\hline $0,00-0,10$ & 5,75 & 5,02 & 6,17 & 4,94 & 6,05 & 4,9 & 5,94 & 4,61 \\
\hline $0,10-0,20$ & 4,29 & 4,28 & 5,00 & 4,41 & 4,39 & 4,58 & 4,35 & 4,26 \\
\hline $0,20-0,40$ & 5,27 & 4,14 & 4,84 & 4,59 & 4,49 & 4,42 & 4,48 & 4,42 \\
\hline
\end{tabular}

Em que: *Diferença significativa antes e após um ano de implantação do Eucalyptus dunnii Maiden dentro de cada tratamento pelo teste t a $5 \%$ de probabilidade de erro.

Semelhantemente ao ocorrido para o $\mathrm{Ca}$, os níveis de $\mathrm{Mg}$ também aumentaram, porém, houve diferença significativa apenas para o espaçamento $3,50 \times 1,75 \mathrm{~m}$, na camada $0,20-0,40 \mathrm{~m}$, e 1,75 x 1,75 m nas camadas 0,10-0,20 m e 0,20-0,40 m.

Essa elevação dos nutrientes no solo após 12 meses da implantação dos povoamentos está relacionada com os efeitos da adubação e da liberação dos nutrientes pela decomposição das gramíneas existentes no campo anteriormente ao plantio. Outro fator contribuinte para este resultado é a baixa demanda de Ca neste estágio inicial, pois há um grande acúmulo do elemento na casca das árvores, cuja demanda ocorrerá em estágios futuros (LEITE, 1998).

Os níveis de P no solo diminuíram 12 meses após a implantação do eucalipto, de forma significativa apenas no espaçamento 1,75 x 1,75 m na primeira camada. Nos demais espaçamentos não houve efeito do plantio de eucalipto nos níveis do nutriente no solo. Esse fato está relacionado com a adubação aplicada e com o espaçamento utilizado. O nutriente aplicado ao solo no espaçamento $1,75 \times 1,75 \mathrm{~m}$ foi totalmente absorvido pelas plantas, devido à elevada densidade populacional e à alta demanda de $\mathrm{P}$ pela espécie no período inicial, enquanto nos demais espaçamentos há uma menor demanda em função do menor número de plantas por área. Dessa forma, há a necessidade de usar maiores doses de fertilizantes fosfatados em espaçamentos mais densos, para não ocorrer degradação química do solo pela depleção dos nutrientes.

Em relação às reduções nos níveis de $\mathrm{P}$ no solo 12 meses após a implantação do eucalipto, tal fato ocorre, pois, as espécies florestais mais jovens exploram as camadas mais superficiais do solo e requererem níveis de P mais elevados, que decrescem com a idade e menores níveis de $\mathrm{K}$, Ca e Mg (BARROS; NEVES; NOVAIS, 2005). Com isso, demonstra-se a significativa redução ocorrida no espaçamento 1,75 x 1,75 m na primeira camada de solo, a qual apresenta um elevado número de indivíduos, o que requer, dessa forma, uma elevada quantidade de nutrientes para suprir essa demanda.

Os níveis de K no solo não sofreram alterações com a instalação de povoamentos de Eucalyptus dunnii. Resultado que corrobora o estudo do efeito da floresta natural, pastagens, vegetação arbustiva, floresta secundária e floresta cultivada nas propriedades do solo realizado por Liu et al. (2002), os quais não observaram diferenças significativas entre os tratamentos nos conteúdos de $\mathrm{P}$ e $\mathrm{K}$ total no solo.

Os níveis de $\mathrm{P}$ para a classe de solo correspondente situam-se na faixa considerada como baixo a muito baixo (SOCIEDADE BRASILEIRA DE CIÊNCIA DO SOLO, 2004), enquanto que, para Novais, Barros e Neves (1986), os valores críticos de manutenção de eucalipto para P, K, Ca e Mg no solo variam de $6,1 \mathrm{mg} \mathrm{dm}^{-3} ; 30 \mathrm{mg} \mathrm{dm}^{-3} ; 0,30 \mathrm{cmol}_{\mathrm{c}} \mathrm{dm}^{-3}$ e $0,05 \mathrm{cmol}_{\mathrm{c}} \mathrm{dm}^{-3}$, respectivamente. No presente estudo, com exceção do $\mathrm{P}$ na segunda e terceira camada e do $\mathrm{Ca}$, os níveis dos nutrientes encontram-se acima do limite crítico, havendo condições nutricionais adequadas para o desenvolvimento do povoamento.

Após 12 meses de implantação do eucalipto, não se observou alteração dos teores de carbono nos 
espaçamentos estudados. Os resultados concordam com Binkley e Resh (1999) que também não observaram alterações significativas nos teores de carbono do solo em florestamento com Eucalyptus aos 32 meses de idade. No entanto, os resultados são contrastantes aos de Paul et al. (2002) e de Turner e Lambert (2000), os quais verificaram redução inicial dos teores de matéria orgânica do solo com o cultivo, sendo tal redução atribuída à maior decomposição dos resíduos orgânicos do solo. Evidências de aumento dos teores de matéria orgânica no solo com o plantio de eucalipto somente após 7 anos de cultivo foram reportados por Mboukou-Kimbatsa, Bernhard-Reversat e Loumeto (1998).

Observa-se que os níveis de $\mathrm{N}$ total do solo também não foram alterados com a instalação dos povoamentos de eucaliptos. Esses resultados são discordantes do padrão observados por Vezzani, Tedesco e Barros (2001), que inferem que, em florestas de eucalipto, há uma elevada demanda de nitrogênio, até a formação da copa das plantas.

Não é possível obter muitas conclusões em relação às alterações ocorridas nas propriedades químicas do solo, nos primeiros 12 meses de instalação do povoamento, sendo necessário um período de tempo maior para que ocorra competição entre as árvores e altere a dinâmica dos nutrientes no sistema. Alguns nutrientes tiveram seus teores aumentados no solo devido ao efeito da adubação aplicada e não à introdução do eucalipto. Enquanto outros, requeridos em maiores quantidades pelas plantas na fase inicial e dependente, do espaçamento, sofreram diminuição no seu teor no solo. Para esses há efeito do adensamento de plantio. Além disso, as manifestações ocorrem ao longo do tempo, sendo que em um curto período de tempo, ainda não é possível observar alterações nas propriedades do solo. Na fase inicial de estabelecimento de uma vegetação divergente à que ocupava o solo anteriormente, ocorrem modificações nos micro-organismos do solo e posteriormente podem levar a modificações na decomposição da matéria orgânica e na ciclagem de nutrientes (ARAÚJO et al., 2010).

\section{CONCLUSÕES}

Após 12 meses de implantação de Eucalyptus dunnii, sob diferentes densidades populacionais em um campo nativo, verificou-se que houve pequenas alterações nas propriedades físicas e químicas de um Argissolo Vermelho-Amarelo Distrófico, com destaque para o aumento da microporosidade e redução na macroporosidade e na estabilidade dos agregados, bem como, a maior elevação do $\mathrm{pH} \mathrm{H}_{2} \mathrm{O}$ e dos teores de $\mathrm{Ca}$, além da redução do $\mathrm{P}$.

\section{REFERÊNCIAS}

ALVARENGA, M. I. N. Propriedades físicas, químicas e biológicas de um Latossolo Vermelho-Escuro em diferentes ecossistemas. 1996. 211 f. (Tese de Doutorado) - Universidade Federal de Lavras, Lavras, 1996.

ARATANI, R. G. et al. Qualidade física de um Latossolo Vermelho acriférrico sob diferentes sistemas de uso e manejo. Revista Brasileira de Ciência do Solo, Viçosa, MG, v. 33, n. 3, p. 677-687, maio/jun. 2009. ARAÚJO, A. S. F. et al. The effect of converting tropical native savanna to Eucalyptus grandis forest on soil microbial biomass. Land degradation \& development, Chichester, v. 21, n. 6, p. 540-545, nov./dec. 2010. BARRETO, A. C. et al. Características químicas e físicas de um solo sob floresta, sistema agroflorestal e pastagem no sul da Bahia. Revista Caatinga, Mossoró, v. 19, n. 4, p. 415-425, out./dez. 2006.

BARROS, N. F.; NEVES, J. C. L.; NOVAIS, R. F. Recomendações de fertilizantes minerais em plantios de eucalipto. IN: GONÇALVES, J. L. M.; BENEDETTI, V. Nutrição e fertilização florestal. Piracicaba: IPEF, 2005.

BAVER, L. D.; GARDNER, W. H.; GARDNER, W. R. Soil structure: classification and genesis. In: BAVER, L. D.; GARDNER, W. H.; GARDNER, W. R (Ed.). Soil Physics. New York: John Wiley, 1972. p. 130-177. BINKLEY, D.; RESH, S. C. Rapid changes in soils following Eucalyptus afforestation in Hawaii. Soil Science Society of America Journal, Madison, v. 63, n. 1, p. 222-225, jan./feb. 1999.

BRADY, N. C. Natureza e propriedades dos solos. 7. ed. Rio de Janeiro: F. Bastos, 1989. 898 p. EMBRAPA. Centro Nacional de Pesquisa de Solos. Manual de métodos de análise de solo. 2. ed. Rio de Janeiro: Embrapa Solos, 1997. 212 p. 
FLORES, C. A. et al. Recuperação da qualidade estrutural, pelo sistema plantio direto, de um Argissolo Vermelho. Ciência Rural, Santa Maria, v. 38, n. 8, p. 2164-2172, nov. 2008.

GAVANDE, S. A. Física del suelos: princípios y aplicaciones. México: Editorial Limusa, 1976. 351 p. GUBIANI, P. I. et al. Método alternativo para a determinação da densidade de partículas do solo: exatidão, precisão e tempo de processamento. Ciência Rural, Santa Maria, v. 36, n. 2, p. 664-668, mar./abr. 2006. KEMPER, W. D.; CHEPIL, W. S. Size distribuition of aggregates. In: BLACK, C. A. Physical and mineralogical methods. Madison: ASA \& SSSA, 1965. p. 495-509.

LEITE, E. P. et al. Acúmulo e distribuição de nutrientes em Eucalyptus grandis sob diferentes densidades populacionais. Revista Brasileira de Ciência do Solo, Viçosa, MG, v. 22, n. 3, p. 419-426, jul./set. 1998. LEROY, B. L. M. et al. The quality of exogenous organic matter: short-term effects on soil physical properties and soil organic matter fractions. Soil Use and Management, Amsterdam, v. 24, n. 2, p. 139-147, jun. 2008.

LIMA, W. P. Impacto ambiental do eucalipto. 2. ed. São Paulo: Ed. da Universidade de São Paulo, 1996. $301 \mathrm{p}$.

LIMA, C. L. R. de et al. Atributos físicos de um Planossolo Háplico sob sistemas de manejo comparados aos do campo nativo. Revista Brasileira de Ciência do Solo, Viçosa, MG, v. 32, n. 5, p. 1849-1855, set./out. 2008.

LIU, S. L. et al. Effects of reforestation and deforestation on soil properties in humid mountainous areas: a case study in Wolong Nature Reserve, Sichuan province, China. Soil Use and Management, Amsterdam, v. 18, n. 4, p. 376-380, dec. 2002.

MARTINS, S. G. et al. Avaliação de atributos físicos de um Latossolo Vermelho distroférrico sob diferentes povoamentos florestais. Cerne, Lavras, v. 8, n. 1, p. 32-41, jan./mar. 2002.

MBOUKOU-KIMBATSA, I. M. C.; BERNHARD-REVERSAT, F.; LOUMETO, J. J. Change in soil macrofauna and vegetation when fastgrowing trees are planted on savana soils. Forest Ecology and Management, Amsterdan, v. 110, n. 1, p. 1-12, oct. 1998.

MELLONI, R. et al. Avaliação da qualidade de solos sob diferentes coberturas florestais e de pastagem no sul de Minas Gerais. Revista Brasileira de Ciência do Solo, Viçosa, MG, v. 32, n. 6, p. 2461-2470, nov./dez. 2008

MORENO, J. A. Clima do Rio Grande do Sul. Porto Alegre: Secretaria da Agricultura, 1961. 73 p.

NOVAIS, R. F.; BARROS, N. F.; NEVES, J. C. L. Interpretação de análise química do solo para o crescimento e desenvolvimento de Eucalyptus spp. - níveis críticos de implantação e de manutenção. Revista Árvore, Viçosa, MG, v. 10, n. 1, p. 105-111, jan./jun. 1986.

PAUL, K. I. et al. Change in soil carbon following afforestation. Forest Ecology and Management, Amsterdam, v. 168, n. 1, p. 241-257, set. 2002.

PREVEDELLO, J. Preparo do solo e crescimento inicial de Eucalyptus grandis Hill ex Maiden. em Argissolo. 2008. 81 f. Dissertação (Mestrado em Engenharia Florestal) - Universidade Federal de Santa Maria, Santa Maria, 2008.

REICHERT, J. M. et al. Variação temporal de propriedades físicas do solo e crescimento radicular de feijoeiro em quatro sistemas de manejo. Pesquisa Agropecuária Brasileira, Brasília, v. 44, p. 310-319, 2009.

REICHERT, J. M.; SUZUKI, L. E. A. S.; REINERT, D. J. Compactação do solo em sistemas agropecuários e florestais: identificação, efeitos, limites críticos e mitigação. In: SOCIEDADE BRASILEIRA DE CIÊNCIA DO SOLO. Tópicos em Ciência do Solo. Viçosa, MG: SBCS, 2007. p. 1-48.

SOCIEDADE BRASILEIRA DE CIÊNCIA DO SOLO. Comissão de Química e Fertilidade do Solo. Manual de adubação e calagem para os estados do Rio Grande do Sul e de Santa Catarina. 10. ed. Porto Alegre: SBCS, 2004. 400 p.

STRECK, E. V. et al. Solos do Rio Grande do Sul. 2. ed. Porto Alegre: EMATER; ASCAR-RS, 2008. 222 p.

SUZUKI, L. E. A. S. Qualidade físico-hídrica de um Argissolo sob floresta e pastagem no sul do Brasil. 2008. 138 f. Tese (Doutorado em Ciências Florestais) - Universidade Federal de Santa Maria, Santa Maria, 2008.

TEDESCO, M. J. et al. Análise de solo, plantas e outros materiais. 2. ed. Porto Alegre: UFRGS, 1995. 
$174 \mathrm{p}$.

TISDALL, J. M.; OADES, J. M. Organic matter and water-stable aggregates in soils. European Journal of Soil Science, v. 33, n. 2, p. 141-161, jun. 1982.

TONINI, H. Crescimento e produção de clones de Eucalyptus saligna Smith, na Depressão Central e Serra do Sudeste, Rio Grande do Sul. 2003. 289 f. Tese (Doutorado em Engenharia Florestal) Universidade Federal de Santa Maria, Santa Maria, 2003.

TURNER, J.; LAMBERT, M. Change in organic carbon in forest plantation soils in eastern Australia. Forest Ecology and Management, Amsterdan, v. 133, n. 3, p. 231-247, aug. 2000.

VEZZANI, F. M.; TEDESCO, M. J.; BARROS, N. F. Alterações dos nutrientes no solo e nas plantas em consórcio de eucalipto e acácia negra. Revista Brasileira de Ciência do Solo, Viçosa, MG, v. 25, n. 1, p. 225-231, jan./mar. 2001.

WOHLENBERG, E. V. et al. Dinâmica da agregação de um solo franco-arenoso em cinco sistemas de culturas em rotação e em sucessão. Revista Brasileira de Ciência do Solo, Viçosa, MG, v. 28, n. 5, p. 891-900, set./out. 2004. 